\title{
Chemical action: what is it, and why does it really matter?
}

\author{
W. John Koolage $\cdot$ Ralph Hall
}

Received: 14 January 2011/Accepted: 18 January 2011/Published online: 4 February 2011

(C) Springer Science+Business Media B.V. 2011

\begin{abstract}
Nanotechnology, as with many technologies before it, places a strain on existing legislation and poses a challenge to all administrative agencies tasked with regulating technology-based products. It is easy to see how statutory schemes become outdated, as our ability to understand and affect the world progresses. In this article, we address the regulatory problems that nanotechnology posses for the Food and Drug Administration's (FDA) classification structure for "drugs" and "devices." The last major modification to these terms was in 1976, with the enactment of the Medical Device Amendments. There are serious practical differences for a classification as a drug or device in terms of time to market and research. Drugs are classified, primarily, as acting by "chemical action." We lay out some legal, philosophic, and scientific tools that serve to provide a useful, as well as legally and scientifically faithful, distinction between drugs and devices for the purpose of regulatory classification. These issues we raise are worth the consideration of anyone who is interested in the regulation of nano-products or other novel technologies.
\end{abstract}

W. J. Koolage

Department of History and Philosophy,

Eastern Michigan University, Ypsilanti, MI 48197, USA

R. Hall ( $\bowtie)$

Law School, University of Minnesota, Minneapolis,

MN 55455, USA

e-mail: hallx171@umn.edu
Keywords Applied philosophy - Chemical action Convergent technology · Device classification · Drug classification - Food and drug law · Physicalism . Nanotechnology $\cdot$ Nanoparticles $\cdot$ Regulation of nanotechnology $\cdot$ Governance

\section{Introduction}

Nanotechnology, as with many technologies before it, places a strain on existing legislation and poses a challenge to all administrative agencies tasked with regulating technology-based products. It is easy to see how statutory schemes become outdated as our ability to understand and affect the world progresses. Developing legislation that will correctly anticipate the technologies and scientific advances of the next 50 years is particularly challenging, and it is unlikely that any piece of legislation could be adequately predictive. Although statutes written many years ago may no longer sufficiently encompass the current state of scientific knowledge or advanced technology, an agency's statutory obligations and broader directives require the agency to regulate the use of such technologies under existing schemes. ${ }^{1}$ This can result in incorrect, inconsistent, or inefficient regulation.

\footnotetext{
${ }^{1}$ The ultimate resolution of how to incorporate new scientific knowledge and new product types into statutory and regulatory systems resides with Congress. Congress often takes many
} 
In this article, we address the regulatory problems that nanotechnology poses for the Food and Drug Administration's (FDA) classification system for "drugs" and "devices." The last major modification to these terms was in 1976, with the enactment of the Medical Device Amendments. Subsequent legislation, regulation, and court decisions have simply refined the core definitional schema created by Congress in 1976. As a result, there is a need, assuming that the current legislative approach remains in place, to clarify how nanotechnologybased products should be classified.

Due to the extremely tiny size of nanoparticles and materials and the novel properties that emerge at this scale, there is very little difference, if any, between the chemistry and physics that occur at the atomic and subatomic level which might, under other circumstances, be used to draw a distinction between drugs and devices. Of course, these same challenges to FDA's current product definition system may also exist for other regulatory systems such as the Toxic Substances Control Act (TSCA) or various environmental regulatory systems. We have chosen to focus on FDA's particular predicament for two reasons: (1) the problem is easier to manage in with a narrower focus and (2) the FDA has a substantial impact on the U.S. public and industries. Roughly 20 cents of every dollar spent by the U.S. consumer are spent on an FDA-regulated product (FDA 2009). Moreover, consumers and patients are dependent on many of these products for their health, requiring an especially careful classification system to properly balance the risks and benefits of new products.

Once we have addressed the need for a proper interpretation of the existing statutory scheme, we will examine some philosophical problems raised by nanotechnology given this scheme. The two primary philosophical issues we will address are physicalism and causality. Physicalism is the idea that, at the end of the day, we are all just physical things. We, just as all things, are made up of fundamental particles held together by basic forces. If this is the case, then what

\section{Footnote 1 continued}

years to act after major scientific changes or advances. The concepts we set forth in this article are directed at the regulatory agencies, which must deal with outdated statutes. However, these concepts could also form the basis for Congressional action to address the impact of new scientific knowledge and new products. distinguishes "bigger" objects, such as molecules (chemical objects) or even human beings (biological objects), and physical objects? When we discovered the physical mechanisms for thunder and lightning, we were in a position to stop talking about Zeus' powers to throw lightning; in the same way, if molecules and humans have as their underlying mechanisms, nothing more than basic forces and fundamental particles, we may also stop talking about chemical or biological objects. That is, if there is no scientifically (or philosophically) sound distinction between these sorts of objects, it is difficult to see how there can be a distinction between drugs and devices. Put yet another way, everything, including traditional "drugs" can be explained by physical (i.e., non-chemical) concepts. If we follow physicalism to its logical end, then traditional drugs are equally devices.

Causation also raises some very interesting issues about nanotechnology-based medical products. This problem is a bit more technical, so we will not say more about it here in the introduction. Resolving these scientific and philosophic issues presents a springboard for providing the basics of an algorithm for properly classifying nanotechnology-based products into either "drug" or "device" regulatory schemes. Perhaps unsurprisingly, under the current regulatory schema, some nanotechnology-based products are "drugs" and others are "devices." 2 Our primary goal throughout the paper will be to provide the tools for FDA regulators, industry, and other interested stakeholders, operating under the existing statutory framework, to deal with the current (and future) assignment of nanotechnology-based products into either the drug or device worlds in a consistent, logical, and predictable manner that satisfies the statutory language and purpose.

\section{Legal scope and import of the drug-device distinction}

While the question of the interpretation of the statutory scheme regarding nanotechnology-based products has obvious philosophical import, it begins

\footnotetext{
${ }^{2}$ For example, nanoparticle silver coatings on implants are viewed as devices, while nanosphere-based drug delivery systems are considered drugs.
} 
as a straightforward legal and practical issue. In this section, we will briefly describe the legal and practical aspects related to our concept of interestnamely what is "chemical action." As discussed in detail below, the difference between drugs and devices is that drugs work via "chemical action," while devices work physically or mechanically.

The key to complex statutory scope and interpretation questions is often found in definitions. The Food Drug and Cosmetic Act (FDCA, 21 USC \$301 et. seq.) is no exception. The definitions contained within the FDCA determine jurisdiction-does the FDCA apply to the product or activity in question? Once jurisdiction is established, the definitions determine regulatory pathways and requirements. Therefore, one must look to key FDCA definitions to determine whether a specific nanotechnologybased product is (1) covered by the FDCA and, if so, (2) what requirements must be satisfied before that product can be marketed (key definitions are generally found in 21 U.S.C. $\$ 321)$.

Generally speaking, medical products, whether therapeutic or diagnostic, are considered drugs (21 U.S.C. $\$ 321(\mathrm{~g})$ ), devices (21 U.S.C. $\$ 321(\mathrm{~h})$ ), or biologics (42 U.S.C. §262(i)). Separate centers within FDA have responsibility and jurisdiction over these different categories of products. ${ }^{3}$ The Center for Drug Evaluation and Research (CDER) regulates drugs, the Center for Biologics Evaluation and Research (CBER) covers biologics, and the Center for Devices and Radiological Health (CDRH) has jurisdiction over medical devices.

While these statutory "details" might be interesting to regulatory specialists, these details make a reallife difference for nanotechnology-based products. Drugs and medical devices follow very different regulatory pathways and have different requirements, different time lines, and different cost structures. The determination of whether a new product is a drug or device has practical, financial, and research-related ramifications.

\footnotetext{
${ }^{3}$ FDA generally does not regulate the practice of medicine. See, for example, 21 USC \$396. Also, dietary supplements and certain foods can make health claims without being considered drugs, devices, or biologics. 21 USC \$343(r) sets forth some requirements for foods making health claims. For our purposes, these exceptions will not be discussed in any detail.
}

The obvious question is whether it makes much of difference whether some product is defined as a drug or as a medical device. The answer is that it makes a tremendous difference. A new drug takes hundreds of millions of dollars, more than a decade, and thousands of clinical trial subjects to get market approval. A new device takes a fraction of that amount of money, time, or clinical trial subjects. While a detailed description of the difference between regulatory processes applicable to drugs and those relevant to medical devices is beyond the scope of this paper, some key differences must be noted.

Any "new drug" 4 must be approved for distribution pursuant to the New Drug Application (NDA) process (21 U.S.C. \$355(a)-(d) and 21 C.F.R. 314). The NDA is based upon extensive preclinical and clinical research, including a three-phase clinical trial process. Clinical trials may total several tens of thousands of patients (21 U.S.C. §355(i) and 21 C.F.R. 312).

Conversely, new medical devices are subject to a three-tier, risk-based regulatory system. Low risk (or Class I) devices need no premarket review by FDAthese devices are subject to "general controls." Medium risk devices (generally Class II device) are cleared for market under the $510(\mathrm{k})$ process, if the device is "substantially equivalent" to a predicate product already legally marketed. Only about $10-15 \%$ of $510(\mathrm{k})$ clearances involve the submission of clinical data.

Class III devices generally must go through the PreMarket Approval (PMA) process. This is conceptually closest to the NDA process and usually includes clinical trials regulated under 21 U.S.C. $\S 360 \mathrm{j}(\mathrm{g})$. Rather than clinical trials lasting for years and involving several tens of thousands of clinical trial subjects, device clinical trials (or IDE studies) are usually much shorter in duration and often involve only hundreds of subjects (21 C.F.R. 812).

In summary, even the most complex devices often take years less to develop and only $10-20 \%$ of the cost of bringing a new drug on the market. The definitional difference between drugs and medical devices thus impacts patient access to new products,

\footnotetext{
4 "New drugs" are defined under 21 U.S.C. $\$ 321(p)$ and, generally speaking, including any new compound or new use of an old compound that came into existence after 1938.
} 
the timing of such access, and cost. Putting a particular nano product into the wrong category risks either excessive regulation or inadequate safety and efficacy review. As such, the chemical action distinction becomes critical.

\section{Statutory background}

To start, one must understand the definition of a "drug" and a "medical device."

In relevant part, drugs are defined in 21 USC $321(\mathrm{~g})$ as:

(1) The term "drug" means

(A) $\ldots$

(B) articles intended for use in the diagnosis, cure, mitigation, treatment, or prevention of disease in man or other animals; and

(C) articles (other than food) intended to affect the structure or any function of the body of man or other animals; and

(D) articles intended for use as a component of any article specified in clause (A), (B), or $(\mathrm{C}) \ldots$

The relevant portions of the definition of a device under 21 USC $\$ 321(\mathrm{~h})$ are:

The term "device" (except when used in paragraph (n) of this section and in sections 301(i), 403(f), 502(c), and 602(c)) means an instrument, apparatus, implement, machine, contrivance, implant, in vitro reagent, or other similar or related article, including any component, part, or accessory, which is

(1) $\ldots$

(2) intended for use in the diagnosis of disease or other conditions, or in the cure, mitigation, treatment, or prevention of disease, in man or other animals, or

(3) intended to affect the structure or any function of the body of man or other animals, and which does not achieve its primary intended purposes through chemical action within or on the body of man or other animals and which is not dependent upon being metabolized for the achievement of its primary intended purposes.

These definitions have several key common features.
First, both are based on the intended use of the product. By using the intended use of the product, rather than what the product actually does in real life, FDA can exercise authority over quack or sham products. An unscrupulous person cannot evade FDA enforcement by arguing that his or her product did not actually treat any disease or otherwise have any therapeutic or diagnostic effect. Both definitions take similar conceptual approaches to this aspect of definitional scope.

Next, both definitions include products that are intended to treat, cure, mitigate, prevent, or diagnose disease. With minor differences, the language covers the same types of intended uses for both drugs and devices. Without the chemical action distinction, discussed below, the same product would be both a drug and a device.

Finally, each definition includes components of the product (and in the case of devices, accessories and parts).

At this point, the same product would easily satisfy both definitions. For example, a cancer drug is an "article" "intended to" "treat or mitigate" a disease (cancer) in a person. An artificial knee is also an "article" "intended to" "treat or mitigate" a disease (arthritis or knee injury in this case) in a person. Does this mean that all drugs are devices and all devices are drugs? Such an overlap violates common sense and norms of statutory construction. It would also subject the same product to vastly different regulatory systems. The key then is to isolate the feature(s) within the statutory schema that differentiate drugs from devices.

The last part of the definition of a device provides the key to separating drugs and devices. As stated in 21 USC \$321(h), a device “does not achieve its primary intended purposes through chemical action within or on the body of man or other animals and which is not dependent upon being metabolized for the achievement of its primary intended purposes." Simplistically, drugs work via "chemical action," while devices work via physics (or mechanically).

\footnotetext{
${ }^{5}$ For our purposes, the metabolism element is not relevant. We do point out the, perhaps inappropriate, use of the "and" connector between the "chemical action" clause and the metabolism clause. In general usage, this has been interpreted as meaning "or."
} 
In most cases, the difference between operating via physical principles and chemical action is clear. Except at the most obtuse level, a stethoscope works via the laws of physics. Under this definition, an artificial knee is clearly a device, as it does not act chemically. The same is true for a heart valve, an artificial joint, or an infusion pump. Drugs, such as oncology products and antidepressants, work via a chemical interaction between the drug and the target cell.

As long as the distinction between chemistry and physics (or mechanical action) is clear, this definitional schema works. Of course, this distinction is precisely what is put to task in cases of nanotechnology-based products.

\section{Possible approaches to the problem}

There are three very reasonable approaches to determining how to classify nanotechnology-based products as drugs or devices in line with the idea that it is "chemical action" that distinguishes them. The first is to look to further legal information (including the history of the related statutes) in an effort to determine the ideas that are central to this classificatory distinction. We call this the legal-historical approach. The second is to look to how such products might be classified in other areas of inquiry. For example, one might ask the chemists how they distinguish these articles. We call this the institutional approach. Third, one might ask whether or not the world draws a distinction between drugs and devices; that is, can we discover, by the proper progress of science, properties of the nanotechnology-based products in question that place them firmly under one or the other classifications? In this section, we will examine these three approaches and discuss why none of them are sufficient for classifying nanotechnology-based products into the existing statutory scheme.

\section{The legal-historical approach}

Under 21 U.S.C. 321(h), the main factors that separate a "device" from a "drug" are that a device: (1) is "an instrument, apparatus, implement, machine, contrivance, implant, in vitro reagent, or other similar or related article, including any component, part, or accessory," (2) that "does not achieve its primary intended purposes through chemical action within or on the body of man," and (3) "is not dependent upon being metabolized for the achievement of its primary intended purposes." Since the concept of being a "primary intended purpose" is relatively well understood legally, we will focus on (2), since it contains the only remaining legally difficult concept-chemical action.

Initially, one must understand the rules of construction or interpretation of complex statutory terms and concepts. In Chevron USA, Inc. v. Natural Resources Defense Council, Inc., 467 U.S. 837 (1984), the Supreme Court set a standard for determining the meaning of the term "stationary source" under the Clean Air Act of 1977. This standard included the use of dictionary definitions, statutory structure, legislative purpose, legislative history, and agency interpretation to determine the meaning of "stationary source." In this way, there is a strong legal tradition that supports attempting to resolve the meaning of the term "chemical action" in the same manner.

Unfortunately, this same approach provides little value in defining the mode of action of most nanotechnology-based products. Part of the problem with dictionary definitions for terms such as "chemical action" is their inability to handle novel cases, especially those generated by emerging technologies and scientific advance. Nanotechnology is a prime example of such a failure. The dictionary definitions for terms like "chemistry" have hardly changed despite leaps in the understanding of chemistry. "Chemical" has consistently been known as a term "of or pertaining to chemistry (Merriam-Webster's Medical Dictionary 2007)." In 1912, a few years after the FDA was created, Webster's New International Dictionary of the English Language defined "chemistry" as "the science that treats of the composition of substances, and of the transformations which they undergo." Today, the same dictionary defines chemistry differently, as "a science that deals with the composition, structure, and properties of substances and of the transformations that they undergo" (Webster's 2002, emphasis added). Though the difference between the two definitions is small, the new terms supposedly reflect fundamental advancements in scientific knowledge, such as Neils Bohr's theory of atomic structure in 1912, and redefinition of the field because of the branching of fields such as 
nuclear physics and quantum chemistry, developed in the mid to late twentieth century. This example demonstrates the problem: dictionary definitions are reactionary only; there is a lag between new ideas and technologies and their dictionary counterparts. As a result, they offer little guidance in the case of genuinely novel technologies or scientific advances.

Other portions of the FDCA may shed light on the meaning of "chemical action" and "metabolism." Particularly, 321(p) defines a "new drug" as "any drug...the composition of which is such that such drug is not generally recognized, among experts...as safe and effective for use under the condition prescribed, recommended, or suggested in the labeling thereof." This definition is dependent on "institutional" conceptions-the beliefs of scientists and experts about the novelty of the drug. This institutional component generates its own problems, which we will consider in the next section.

Additionally, to clarify the "new drug" definition, FDA required that a new drug must be "new chemical entity," which is a term of particular interest because it may lead to a meaning of "chemical action." The FDA has previously defined "new chemical entity" to mean a drug that contains no "active moiety" that has previously been approved by FDA. "Active moiety," in turn, means "the molecule or ion ... or other noncovalent derivative... of the molecule, responsible for the physiological or pharmacological action of the drug substance" (21 C.F.R. 314.108). One conclusion may be drawn from this definition: the FDA has previously asserted that the term chemical in the new drug context involves "molecules" or "ions" that are "responsible for" the action of the drug. Though this definition of chemical within the new drug context is not binding on the Agency in making initial definitional decisions, it provides one persuasive (though incomplete) interpretation of "chemical action." Our own account will make use of this idea. However, since "new drug" is a subset of "drug," it does not follow that moiety is sufficient to generate a distinction between "drugs" and "devices"-it merely identifies a subset of the "drug" class.

FDA has been given broad discretion to apply its regulations in a manner to promote public health. Courts have interpreted the FDCA to have a "remedial purpose" (Bacto-Unidisk 1969). The FDA was originally created to "effectuate the congressional purpose of protecting the public" from unsafe drugs, or "quack" devices that made untruthful promises to consumers or were otherwise unsafe (understanding that safety is a relative term). In view of this remedial purpose, the Bacto-Unidisk court's reasoning suggests that, among the competing considerations in defining chemical action, the greatest value should be assigned to factors that "protect the public." Thus, as detailed above, legislative intent or history only tells us that there is a desire to protect the public, not the details of defining nanoproducts. This is of little value in classifying "drugs" and "devices," since both are regulated with public safety in mind. However, it may be of some help in hopelessly ambiguous cases: if we should find an article which cannot be properly classified as to its nature as a drug or device, we should place it in the class that would lead to the greatest consumer protection.

When the language or application of a statute is unclear, courts and administrative agencies commonly turn to legislative history for guidance (BactoUnidisk 1969). In particular, understanding the legislative history behind major changes to the definitional structure of the FDCA made in 1938, 1976, and 1990 may help explain differences between "drugs" and "devices" and the crucial "chemical action" distinction.

\section{The 1938 amendments}

Before the 1938 changes to the Pure Food and Drug Act of 1906, FDA had little ability to control therapeutic claims made for drugs and devices (Merrill 1996). Medical devices were generally simple devices such as bandages and scalpels that presented few technological issues and whose use was generally obvious to physicians and patients (FDA 1997).

In 1938, the Food and Drug Act defined drugs narrowly, with no specific mention of devices. Specially, drugs were defined as "all medicines and preparations recognized in the United States Pharmacopoeia or National Formulary for internal or external use, and any substance or mixture of substances intended to be used for the cure, mitigation, or prevention of disease of either man or other animals" (Bacto-Unidisk 1969, pp. 793-794).

This narrow 1906 definition of drugs left "quack" devices out of the legal control of FDA, leaving 
consumers unprotected from products such as "radium belts" and "slenderizers" (Senate Report No. 361 1935). This led Senator Copeland of New York, the sponsor of the 1938 FDCA, to include "all substances, preparations, and devices intended for use in the cure, mitigation, treatment, or prevention of disease in man" into the definition of "drug" (Senate Report No. 493 1934). The inclusion of "devices" in the definition of "drugs" sparked a lively debate in the Senate, with Senator Clark calling the treatment of a "purely mechanical device" as a drug in "law and in logic...a palpable absurdity" (Congressional Record 1935, p. 4811). The final bill proposed a definition of "device" paralleling the definition of "drug," even though both were regulated in the same manner at the time.

In Bacto-Unidisk, the Supreme Court interpreted the legislative history behind this parallel definitional structure and concluded that the differences between drugs and devices were "provided for semantic reasons only" (Bacto-Unidisk 1969, p. 797). The Court used this reasoning to support a broadly inclusive definition of "drugs" and held that an antibiotic sensitivity test disc used in laboratories was a "drug" within the meaning of $\$ 321$.

However, the approach of Congress to regulate devices in "terms that accurately describe [devices]" rather than as drugs may be interpreted differently (Congressional Record 1935, p. 4812). The debate in the Senate suggests that Senator Clark and his colleagues saw an innate difference between mechanical operation of devices and "drugs." Despite arguably treating drugs and devices equally at this time, Congress may have emphasized the definitional difference because it sought to avoid future regulatory problems arising from legally equating two entities with innate differences. In addition, it is plausible that Congress' later amendments in 1976, essentially overturning the specific holding of BactoUnidisk, reaffirm the definitional and regulatory differences between "drug" status and "device" status. Nonetheless, the legislative history from the 1938 Amendments does not clearly elucidate Congress' intent surrounding the specific differences between "drugs" and "devices," apart from the few and incomplete examples mentioned in floor debates. Under the original 1938 approach and Bacto-Unidisk, a nano-product could be regulated as a drug regardless of its mode of action.
It is also instructive to remember the state of scientific knowledge about the mechanisms of chemical actions in the mid 1930s. The general consensus among experts is that physics and chemistry became clearly distinct scientific subjects in the 1830s (Nye 1993). When the FDCA was being adopted, scholars were just starting to challenge the distinctions between chemistry and physics (Nye 1993). Thus, the difference between a mechanical device and a chemical drug was much clearer in the 1930s than it is today, despite significant progress in the sciences since then.

The medical device amendments of 1976

The 1938 Act only gave FDA the authority to remove misbranded medical devices from the market, not the authority to review medical devices before entering the market. Subsequent amendments in 1962 established a premarket review and approval process for drug efficacy, but the amendments did not apply to devices. ${ }^{6}$ This reinforces the idea that Congress viewed devices and drugs differently. This gap in regulatory requirements for drugs and devices set the stage for Bacto-Unidisk, which gave FDA greater discretion in regulating some items commonly thought to be devices as drugs, in order to place greater controls on these devices.

Congress adopted the Medical Device Amendments of 1976 (MDA) in response to the confusion between drugs and devices, the findings of the Cooper Commission suggesting that medical devices caused thousands of deaths and several incidents involving pacemaker failures and the Dalkon Shield intrauterine device (Hutt et al. 2007). Aside from creating a tiered approval process for devices to ensure greater consumer protection from the riskiest devices, Congress refined the boundaries between drugs and devices. According to the amended statute, a device does not achieve any of its "principal intended purposes through chemical action and...is not dependent upon being metabolized," and a "drug" does not include "devices or their components." The reasoning behind this definition was discussed in Senate debates:

\footnotetext{
${ }^{6}$ There was some pending device-specific legislation around the same time as the 1962 drug amendments. Perhaps because of the challenge posed by thalidomide, the drug amendments were passed, but the proposed device amendments languished.
} 
Drugs, which are basically chemical entities, are consumed on a short-term individual dosage basis and are changed or destroyed in the body. Devices, on the other hand, which involve all the physical sciences plus all the divisions of the biological sciences, are used on an individual product basis for a short time or for the life time of the patient, and are usually intended to be as inert as possible (Congressional Records 1975).

Note that Congress is linking drugs with chemicals and devices with the "physical sciences." This difference subsequently becomes the fundamental basis for differentiating between drugs and devices that operate at the nano-scale.

\section{Safe medical device act of 1990}

In 1990, Congress made a minor modification to the definitions of drugs and devices in 21 USC 321(g) and (h). These amendments altered (1) in paragraph (g)(1) by striking out "but does not include devices or their components, parts, or accessories," and (2) in paragraph (h)(3) by striking out "any of its principal" and inserting instead "its primary."

The committee report in the Senate contains the only discussion of these particular modifications, stating, "The definitions of 'drug' and 'device' have been slightly altered to accommodate the principle of section 20 [addressing Combination Products]" (Senate Report No. 513 1990, pp. 30-31). Thus, the modifications were likely intended to conform to the terminology established in the combination products regime and to allow FDA to regulate a combination product with both device and drug components as a drug, if it so chose. These amendments were likely not intended to clarify the meanings of "drugs" and "devices." Without these changes, a combination product that included drug and device components could not be regulated as a drug, even if the drug portion was the "principal mode of action" (PMOA). In developing the combination product regulatory structure, Congress retained the core definitions and allowed the regulatory structure responsible for the primary mode of action to handle the regulatory needs. This concept also reinforces the concept that the method or mode of action of the article is a key differentiating factor between drugs and devices.
Combining the Chevron doctrine, statutory language, and legislative history, there are two main conclusions: (1) legal analysis does not completely resolve the problem because dictionary definitions are unclear and the legislative history is not dispositive, but (2) it does give us a starting point for understanding chemical action and metabolism from general statutory purpose and statutory structure: "chemical" means something to do with molecules and ions, and we should err on the side of greater "public protection" in view of the larger statutory purpose.

It is worth noting that if Congress had explicitly defined "chemical action," that definition must be used to assign nanoproducts into the drug or device worlds. This is so even if the Congressional definition made no sense to scientists. Given that FDA is a creature of statute and is completely reliant on statute for its existence and power, Congress can make whatever definitional assignments it chooses. Unfortunately, the statutory language and legislative history simply does not answer the question. As such, other analytical approaches must also be considered.

\section{The institutional approach}

In light of the indeterminacy left by legal analysis, we will broaden our search for a precise account of chemical action, and, in turn, "drug." There is a very simple strategy for determining whether some article operates by chemical action, rather than by mechanical action. This strategy faces serious problems in our current scientific environment. Understanding what is wrong with this strategy makes clear the need for a more fine-grained approach, which we offer in section the next section.

We call this strategy the institutional approach. On this account, we determine whether an article operates by chemical action by asking which department at the local university or similar "expert" institution is the one that developed the article or the theory by which the article was developed. In the past, the institutional approach was very effective. For example, chemists and chemical engineers were the owners of such articles as plastic or detergent, whereas physicists and physical engineers were the owners such articles as the wheel or the clock. 
A problem arises due to the conventional nature of the institutional approach: the conventions we once used for carving up the sciences are now outmoded and obsolete. To see this problem clearly, we only need to look to articles that are convergent technologies.

Convergent technologies, especially ones that are really, really tiny, and with really, really big surface areas, break traditional boundaries-they are designed and understood by a number of theoretical and applied sciences. For example, designing a particular object may require theorists from several fields, like cellular biology, quantum mechanics, and computer science, as well as engineers from chemical engineering, mechanical engineering, and so on. To which area, specialization, or science does this new nanotechnology belong? Consider the recent developments in nano-origami. Nano-origami is the folding of nano-scale materials into three-dimensional shapes. One could design a tiny box, complete with a latch that can be opened with a magnetic current. Such a box could be used to deliver and release antibacterials, for example. Would such a nano-box with a medical payload fall under the purview of chemical or physical engineering? Would it use only physical theories, which are the purview of physicists? Or, would it require a good deal of chemical, medical, and biological theories, as well? We take it that there is no simple and obviously non-arbitrary answer to these questions. It follows that its uses, functioning, or limitations are not "owned" by any one theory or body of theories within any of the traditional divisions of the sciences.

The institutional approach depends on decades old differentiation between chemistry and physics (or the mechanical arts). This institutional approach was current, and the state of the art across the scientific world when the drug/device regulatory system was created in the mid to late 1930s. As we saw earlier, there is a need to update these ideas. That is, the institutional approach at the very least needs an upgrade.

\section{The actuality approach}

A third and more bottom-up approach than the institutional approach is available. We call this approach the actuality approach. Rather than appeal to rather outdated and arbitrary conventions of days gone by, one might think that the world itself and current scientific knowledge will tell us whether something is chemical or not. In fact, it is quite reasonable to think that certain theories, like chemistry, are a response to our need to predict certain kinds of actual phenomena. Consequently, whether a particular phenomenon belongs to a certain science depends not on mere institutional conventions; rather, it depends on the concepts required to predict and understand the phenomenon. That is, something is a chemical action (a kind of phenomenon) if we need concepts from our best theories of chemistry to predict and understand that action. Put another way, if chemistry is a response to the way the world is, then we have discovered chemical properties of the world: the science of chemistry is required to understand some phenomena.

One might think that the actuality approach also faces a serious problem in light of convergent technologies. It is not so obvious to us that it does - if theories of chemistry are employed in the development and understanding of nano-technology, then there is some sense in which the end product (does or does not) operate by chemical action. The problem is now to say what counts as a chemical theory, since we cannot identify such a theory by mere appeal to the institutional approach. This points us to a second problem well known to philosophers and generated by physicalism. ${ }^{7}$

Physicalism is the well-accepted belief that, at the bottom, all phenomena are physical phenomena. That is, almost all of the hard sciences accept that the objects and processes they study are literally physical objects and processes that can be explained or described physically (e.g., using theories of physics). While it may be useful to talk about organisms or neurotransmitters, in the end these, like all other things, are made up of particles and their relations are described in the language of physics. This assumption makes trouble for the actuality approach: chemical concepts (and theories) differ from physical concepts (and theories) only in terms of their utility. The phenomena understood and predicted by chemical theories could be (though it may be very difficult and time consuming) captured entirely by physical

\footnotetext{
7 Physicalism is a term that can be traced back to Otto Neurath (2000).
} 
theories, if this well-accepted assumption is correct. Thus, the actuality approach faces a very different problem than the institutional approach: chemical theories are just different from physical theories in practice-there is nothing about the world itself (other than our need to predict in a relatively accurate and effective manner) that distinguishes the two theories. Chemistry is simply a wholly included subset of physics. In essence, it is believed that we could re-write all the chemical theories in terms of our physical theories.

As can be seen, none of these three basic approaches provides a clear, concise, or predictable answer to the question about whether or not an article has as a primary mode of action one that is chemical rather than physical. As such, we now proceed to offer an approach that seeks to be true to the statutory language and legislative intent and also is true to science and logic.

\section{The four pillars of chemical action}

In this section, we present the four key ideas to understanding chemical action in the FDA context. These pillars of chemical action are aimed at balancing and resolving the problems with the legal-historical approach, the institutional approach, and the actuality approach. The goal is to produce an account of "chemical action" that meets the legal and regulatory needs, while also being sensitive to the scientific, philosophical, and legal questions articles such as nanotechnologies pose. In this section, we will discuss the pillars fairly generally. When these pillars are applied as principles they become useful for regulators. In the next section, we will utilize these four pillars and the existing statutory structure to provide an "algorithm" to aid in the regulation of articles (especially nanotechnologies) in terms of their nature as either drugs or devices. That is, when the principles are applied to individual cases, they serve to determine if the case in question is one where a distinctly chemical, rather than physical, action is the primary mode of action.

The four pillars of chemical action are as follows: (1) the chemicality principle, (2) the causality principle, (3) the singularity principle, and (4) the locality principle. The first principle is used to target the philosophical problem raised by physicalism, which is especially salient in the case of nanotechnologies. The other three principles are designed to help resolve problems raised by convergent technologies and the multiplicity of events that occur throughout a treatment.

\section{The chemicality principle}

By way of quick review, physicalism is just the idea that all things are, at the bottom, just particles and forces-just as physics describes. For our purposes, it might be easiest to imagine it this way: if a chemical bond can be completely described using quantum mechanics (one of our most predictively successful theories of physics), then in what sense is it a chemical thing and not merely a physical thing? The mode of action can be completely and accurately described using the concepts, theories, and language of physics. The physicalists suggest that this demonstrates that the process of chemical bonding is not some special new thing; rather, it is just another action of the simple particles and forces described by physics. That is, physicalism suggests that all actions, whether psychological, biological, or chemical are just (sometimes very complex) physical actions. As a result, there can be no difference between drugs and devices, as described in the legislation, because there is no real such thing as "chemical action."

Our chemicality principle suggests that there is a difference worth noting that supports a non-arbitrary distinction between chemical things and physical things. There is a noteworthy difference between ontology and explanation. Ontologically speaking, all things might well be physical things, as physicalism suggests. However, it does not follow that it is best to explain all phenomena using our best physical theories. Explanations can, and should, make use of concepts that are not strictly "at the bottom." That is, even though you and I are simply particles operated on by physical forces (ontologically speaking), it does not follow that best explanation of your reading of or my typing of this paper is best explained using all and only physical concepts, such as particles and forces. Consider a couple of examples: Fodor's Lunch and Lotka-Voltera equations.

Jerry Fodor argues that folk psychology, our everyday theory of the behaviors of ourselves and others, does an excellent job of explaining everyday 
interactions. His arguments suggest the following sort of story of Fodor's Lunch (Fodor 1990). Imagine Jerry Fodor tells you that he will meet you for lunch to discuss his most recent work. The next morning you awake, and you are interested in predicting whether Jerry will meet you; you are also interested in explaining why you think he will meet you. If you were to proceed using the ontologically most basic things (forces and particles), you are in for a very long (if not impossibly complex) set of calculations. Not only that, it is not at all clear that you will have explained why he ultimately met you, why you believed he would, or any of the other steps in between. In fact, prediction an explanation of this event, his meeting you for lunch, are not only more easily, but perhaps best, given in terms of our everyday psychology-using concepts such as intention, promise keeping, memory, and so on. Fodor's Lunch suggests that there are good psychological explanations that we have reason to keep and accept, even though the world is only made up of particles and forces.

Another good example comes from work by Elliott Sober (2000) and makes use of the Lotka-Volterra equations in biology. The Lotka-Volterra equations provide us with a model that predicts predator-prey relationships in a particular environment. Predation, for example, is not obviously nor easily reduced to physical concepts. Furthermore, the model correctly (truthfully) predicts the number of predators in an environment. Thus, by way of explanation, the LotkaVolterra equations (and the related concepts) explain the number of predators by the number of prey animals. Thus, we have a biological explanation that is an accurate predictor and also potentially true. This is a nice example, because it shows that we do not need to reduce to the "at bottom" concepts for a truthful explanation (and also prediction) of the way the world is. It also very nicely shows that there would be something lost by reducing to the "at bottom" concepts (like particles and force)-we would not be able to explain why there are the number of predators there are in a particular environment. For example, the equations explain and predict why predator populations increase and by how much when there is an explosion in the prey population. This truth finds no home in the language of particles and forces, yet properly describes a state of the world.
Our chemicality principle turns on this idea that there is a difference between ontology (what things there are) and explanation (how we properly and predicatively describe the things there are). As with the two examples above, there are chemical concepts that do similar work in explanation and prediction. Even if there are physical (force and particle) correlates of these concepts, there would still be a chemical explanation. As of the current state of chemistry and physics, there are some chemical concepts that both do a lot of explanatory work and also are irreducible to physical explanation. According to Lombardi and Labarca (2005), molecular shape, chemical bond, and orbital are not describable in terms of Quantum Mechanics-that is, they have no physical explanatory analog. Thus, we propose that any explanation of the operation of an article that requires a chemical explanation (or could be given one with some gain in information-as the concept of predation in the biological case) counts as a chemical explanation. This is half of the puzzle: we know what "chemical" means in chemical action. Further, it resolves the trouble raised by physicalism by appealing to information provided by a chemical explanation rather than simple "at bottom" account of the physical nature of things.

\section{The causality principle}

Now that we have addressed what it means to be "chemical" in a chemical mode of action, our next task is to say what it is to be a mode of action. Our analysis of mode of action is to treat it as a cause. That is, an article has a primary mode of action that is chemical if and only if there is a chemical explanation of the article's interaction at the site of treatment whereby the article is described such that it is casually efficacious. In the next three subsections, we will spell out the details of this set of ideas.

One theory of causation that fits closely with several primary legal ideas of causation is probabilistic causation. Probabilistic causation, at its heart, is the idea that a cause is anything that increases the probability of some outcome. For example, sticking your finger in the light socket is a cause of electrocution, even if sometimes one is not electrocuted by so doing. Eells (1991) describes a fairly comprehensive theory of probabilistic causation. We 
will use the basics of his theory to help us understand what it is to be a mode of action and further to understand what it is to be a primary mode of action.

A probabilistic framework for causation sits nicely with a number of legal notions. Consider one example from tort law. Imagine there is an accident in which a car hits a median. If driver error is not the cause of the accident, one can imagine an injured driver suing the car manufacturer, the tire manufacturer, and the city that constructed and maintains that stretch of road. This makes a good deal of sense on the probabilistic view because if it was not the driver who was at fault, then things like tires, accelerators, steering columns, and road maintenance and design are all factors that increase or decrease the probability of such an accident. In fact, it is perfectly possible that all of these things, jointly or severally, are causes of the accident.

An obvious problem arises for providing an analysis of chemical action in the probabilistic framework: we are interested in whether or not the article operates primarily by chemical action, not merely whether there is a chemical explanation of a cause of treatment. Let us call this the problem of relata. In the probabilistic framework, $x$ causes $y$ if and only if $x$ increases the probability of $y$ (in fixed framework $z$ ). $x, y$, and $z$ are known as relata. If we want to know whether some article chemically causes some treatment, we will have to know what $x, y$, and $z$ are. To resolve these questions, we will need our last two principles: the singularity principle and the locality principle.

\section{The singularity principle and the locality principle}

For the sake of example, let us imagine a new anticancer article. This article is introduced orally and takes some time to break down before it is taken up into the bloodstream and produces any tumor reduction results. As it breaks down, part of the article resolves into high levels of calcium. This calcium, in turn, produces an increase in probability of constipation in humans. Is this article a drug? It seems that it may be. First, it produces an increase in probability of two effects: tumor reduction and constipation. Second, the constipation is a result of a chemical change that is the result of the introduction of the article (the additional production of calcium in the system).
While this is a completely hypothetical example, it serves to show why we need to resolve the problem of relata. Recall that for an article to be considered a drug, it must have a primary mode of action that is chemical. Is the side effect of production of calcium the sort of thing that would be considered a primary mode of action? Surely not.

In our probabilistic framework, it is relatively easy to resolve part of the problem of relata. For $x$ to cause $y$ in framework $z, x$ must increase the probability of $y$ with respect to $z$. $y$ and $z$ are relatively easy to understand, so we will begin there. When the FDA evaluates new drugs and devices, they are partly characterized by their primary intended purpose. That is, they are described in terms of what they are supposed to do. This idea of primary intended purpose will serve to identify the $y$ and $z$ relata. $y$ is the proposed treatment. So, we are really asking: does the chemical action of the article increase the probability of the treatment $(y)$ ? Returning to our example, the constipation is not the primary intended purpose of the article, so the chemical change that produces this effect is irrelevant for our classificatory purposes. Something cannot be deemed a drug because of some side effect; it must be the intended effect that is used in the analysis.

$z$ is also relatively easy to understand; $z$ is the entire state of the world. The purpose of control studies is to show that altering a single part of the environment produces an increase in the probability of some effect (treatment). Control studies are good because they provide a way of showing that no other factor is the cause of the positive outcome other than the single altered item of interest. Thus, returning to our cancer treatment, we want to know if the introduction of the article and not some other thing, such as placebo effect, produces the tumor reduction. If we were able to hold fixed all the entire state of the world except for the chemical action of the article, we would know for sure that it was the article that produced the treatment.

At first, $x$ appears to be relatively easy to identify, as well; it is the article in question. However, it is not as easy as that, since it must be $x$ 's chemical properties that increase the probability of the effect and it must be primarily these-otherwise we cannot say if the article (x)'s primary mode of action is chemical. To resolve this remaining problem, we will need the singularity principle and the locality principle. 
The singularity principle demands that we identify a result of the article that produces the treatment. Recall, the legal notion of causation is broad. In the car accident, it is very reasonable to say the cause of the accident was the tires, the road, and the design of the car; it is also reasonable to say that each of these is a cause in the accident. This broad concept must be narrowed if we are to determine whether it is a chemical action of the article that produces the treatment. This is akin to asking whether the tires were, in fact, a probability increaser of the accident. So, if some studies show that our anti-cancer article is associated with tumor reduction, the singularity principle demands that we identify the article as a probability increaser of the tumor reduction. When paired with the chemicality principle, there must be a chemical explanation of the article's increase in probability of the treatment. That is, the article's action must involve change in molecular shape, chemical bonding, or some other factor so long as it can be described using standard concepts in chemistry on its way to increasing the probability of the treatment.

To see how the locality principle comes into play, we can return to our hypothetical anti-cancer article. Imagine that this article, when introduced to the body, does increase the probability of tumor reduction. Further, when ingested, there is a change in the article's molecular shape that produces the constipation. In addition, there is a further chemical change in the article when it is excreted from the body. These two chemical changes occur upstream and downstream of the treatment, and, as a result, these chemical changes are not salient to classifying the article as having a primary mode of action that is chemical and should be ignored. This problem is resolved by the locality principle. This principle demands that we look to the treatment site to determine whether or not the article increases the probability of tumor reduction by chemical action. Recall that the treatment is determined by the primary intended purpose of the article, here tumor reduction. Thus, to avoid being distracted by chemical changes that occur upstream or downstream of the treatment, we look to the tumor areas to see if there is a chemical change in the article that causes the tumor reduction.

The four principles work together to provide us with an account of chemical action as it pertains to the classification of articles as either drugs or devices. Put simply, an article is a drug if it meets the following description:

There must exist an explanation in terms of chemical concepts (chemicality principle) whereby the article in question (the singularity principle), by undergoing some chemical change, increases the probability (the causality principle) of the treatment, at the site of treatment (the locality principle).

In the next section, we introduce an algorithm intended to guide regulators in determining whether an article fits the above description. Later we will provide two "real life" examples that could be resolved using our algorithm.

\section{The chemical action algorithm}

Definitional aspects of algorithm: the centrality of the locality principle

1. We begin by determining primary intended purpose, in combination with the locality and singularity principles. Primary intended purpose is determined by first determining the therapeutic effect (treatment). With that in hand, we can identify the primary causal chain. This is the sequence of events that is necessary for, or creates the greatest likelihood, producing the therapeutic effect.

2. We determine the mode of action by reviewing the intended therapy at the "locality" of the treatment. The relevant assessment is conducted at the first interaction between the article and the local therapy site on the primary causal chain.

3. There are often multiple causes and effects on, near, or related to the chain from introduction of the article to final treatment effect. The locality point of interest includes the following cause and effect relationships:

a. The cause we are interested in is the action at the site where the article interacts with the body to achieve the intended purpose.

b. The effect we are interested in is the primary or triggering therapeutic effect the article has on the body on the chain to treatment. [We 
define "therapy" to include mitigation, prevention, affecting structure, etc.]

4. Looking through this locality lens is justified because "primary intended purposes" yields more consistent classifications of products when compared to looking at the products "as a whole."

Definitional aspects of algorithm: chemical action

5. "Chemical action" means:

1. A chemical change in the article at the site of treatment (locality),

2. at least one of the chemical changes belonging to the article (singularity),

3. in which the chemical change leads to (i.e. causes) the therapeutic effect (causality), and

4. the description of the interaction is consistent with current Chemistry, given the context of the treatment (chemicality).

6. Any change in the shape or structure of the article or target molecule or atom need not be permanent.

7. Ancillary or subsequent changes in molecular or atomic structure which are not the basis upon which the therapeutic action is achieved or that occur at some point other than the locality shall not be relevant to defining chemical action.

Definitional aspects of algorithm: metabolism

8. Again, we first find the primary causal chain with respect to the primary intended purpose that causes the intended therapeutic effect. Then we determine whether metabolism takes place.

9. Metabolism means any necessary molecular modification to the article, achieved via the biological, digestive, or respiratory processes of the body, required for the article to achieve its intended purposes. Metabolism occurring after the therapeutic affect is achieved, or that is not part of the causal chain effectuating treatment, is not relevant to classification.
Other definitional aspects

10. The cause or risk of unintended or adverse events is not relevant to the analysis. Those risks are addressed by the respective regulatory systems. Thus, if glue (a device) causes or has the risk to cause chemical burns, that is for the device regulatory system to identify and mitigate. The fact of chemical burns does not make glue a drug.

11. Special statutory definitions override this algorithm. For example, products meeting the definition of a "biologic" under the PHSA, tissue products under 21 C.F.R .1270 and 1271, and in vitro diagnostic reagents do not go through this algorithm.

Summary

12. Thus, to determine the classification of an article, one first determines the most probable chain to effectuate the therapeutic result. Next, one assesses whether there is "chemical action" at the locality using the definition of chemical action above. The "chemical action" must be the primary or triggering event (cause) in the therapeutic chain. If the article satisfies these conditions, then it operates via chemical action.

Next, one looks to see if metabolism, as defined above, is required for the article to achieve the intended affect at the relevant locality.

If the article in question satisfies neither the definition of chemical action nor the definition of metabolism, then it may be a device.

\section{Cases: silver nanoparticles and nanoparticle sunscreen}

To see how regulators may use this algorithm, we will apply it to a pair of nanotechnologies already in use: silver nanoparticles used as an anti-microbial and zinc nanoparticles used in sunscreen. Somewhat surprisingly, we argue that silver nanoparticles should be classified as a drug, whereas the zinc nanoparticles should be classified as a device. While 
these are not particularly involved nanotechnologies, they are fairly easy to understand and display the way in which the algorithm works. Further, neither are metabolized, so they are particularly useful in highlighting the analysis of chemical action we are interested in conveying.

Silver nanoparticles are used as an anti-microbial. Silver at the nanoscale exhibits some properties with respect to killing bacteria that bulk silver does not. Though the exact mechanism is not well understood (Rai et al. 2009; Singh et al. 2008), we know that the introduction of silver nanoparticles decreases the survival and reproduction of bacteria that can and do cause infections. According to the best available hypotheses, silver nanoparticles, due to their small size and large surface area, release silver ions easily and in great number. These ions work on bacteria in two ways: (1) they bond to the cell wall of bacteria inhibiting respiration and (2) they disrupt the reproductive mechanisms of the bacteria.

The primary intended purpose of the nanoparticle silver is to act as an antimicrobial. This tells us how to determine the therapeutic effect-they decrease or prevent the bacteria that cause infection. Using our locality lens, we need to look at where the silver interacts with the body producing this therapeutic effect. As we have seen, the silver releases silver ions at the treatment site-the bacteria itself or the infection site (as well as elsewhere, but we have been clear we are only interested in changes in the article at the site of treatment). These silver ions go to work preventing the proliferation of and survival of the bacteria. Now, all that remains is to determine whether the silver is metabolized (which it is not) or if it operates by chemical action.

The silver nanoparticles operate by chemical action when used as an antimicrobial. The silver undergoes a change in molecular shape. Additionally, there is the production of silver ions $(\mathrm{Ag}+$ that escapes from the weaker bonds on the large surface small size $\mathrm{Ag}$ ) by breaking of chemical bonds. It is this chemical change that leads to the antimicrobial treatment. Thus, at the site of treatment (therapeutic effect), there is a change in the article, the silver nanoparticle, where it is specifically this change in the article that leads to the reduction and prevention of bacterial infection; further, the explanation of this interaction (including, most importantly, the change in the silver) is well explained by our best available chemical theories. In fact, it is the chemical properties of the sliver at the nanoscale that are our best explanation of the antimicrobial effects of the silver. Thus, it meets all four of our principles (and the more rigorous algorithmic version) to be classified as a drug-its primary mode of operation is chemical action.

We can contrast the silver nanoparticles used as an antimicrobial with the use of zinc nanoparticles in sunscreen. The FDA has chosen to regulate sunscreen as a cosmetic, which has a separate set of regulatory rules; however, we will use it here for purely illustrative purposes. Zinc has been a staple ingredient of sunscreen for many years now. The zinc produces the effect of making the sunscreen look like a white paste on the skin. By making the zinc particles in the sunscreen nanoscale, this effect can be greatly reduced, rendering the sunscreen nearly transparent while, at the same time, retaining the zinc's light reflecting properties.

In the case of sunscreen, the nanoparticles' primary intended purpose is the protection of the skin from harmful light from the sun. The treatment site is the surface of the skin. We then look at how the article, the zinc nanoparticles, interacts with the body to produce the effect. Sunscreen works the same way a porous umbrella might work-it blocks a good deal of the light from striking the skin. There is no chemical explanation required: the sunscreen is a purely mechanical barrier between your skin and the sun. Further, there is no chemical change in the zinc required to explain the sun protecting effect. Thus, sunscreen should not be classified as a drug, as its primary mode of action is not chemical.

\section{Remaining issue: non-functional articles}

One class of products that causes a problem for our algorithm is the class of products that are treatment inert. Products that produce no actual treatment defy classification according to our algorithm. The FDA does, and should, regulate products that merely claim to have medical properties. Consider snake oil. Since such a product claims to produce treatment effects, even though it does not, it does have a "primary intended purpose" that we can easily understand. However, since it has no actual "mode of action," our algorithm cannot serve to classify such a product. 
This disquieting result occurs because our algorithm identifies articles as "chemical" based on whether there is a chemical explanation of the action at the treatment site. Where there is no action, there can be no chemical action.

Since public safety is clearly at issue in the statutory structure, we believe that products that are inert with respect to treatment should still be regulated. This problem needs to be addressed further, but one suggestion is to simply dump this class of objects in one of the current classifications, whether drug, device, or combination. FDA is tasked with regulating articles with public safety in mind, so, in a relatively strong sense, it does not matter into which classification inert articles fall. Current regulations are intended to protect the public from defective devices and dangerous drugs. We can see no obvious and non-arbitrary way of assigning inert articles to one class or another at this time.

For those interested in pursuing this question, here is our current work to include therapeutically inert articles in our algorithm.

\section{Non functional articles}

1. In the event that the article in question has no actual effect, it may still be regulated based on intended use. In such a case, consistent with case law on intended use, the objective intent of the manufacturer, usually evidenced by statements from the manufacturer, will be used to determine its "mode of action" for classification purposes. This will require us to assume that a nonefficacious or even inert article functions as the objective intent of the manufacturer would require if it did work.

Example 1 A company pitches a magnetic cure for arthritis. The magnets do not in fact produce the suggested theraputic effect. It would be regulated as a device as, if it worked, it would work via nonchemical means

Example 2 A company sells a pill claiming that it cures cancer. The pill does not do anything. This would be regulated as a drug because, if it worked, it would have worked chemically

\section{Conclusion}

New technologies often present challenges to statutory schemes developed years ago (and usually by non-scientists). Nanotechnology poses just such a challenge to FDA. Nano products used for therapeutic purposes must be correctly classified as drugs or devices in order to effectuate the statutory purpose. Further, a good deal of money and time can be at stake in the classification. The classification difference relies on drugs operating "chemically" and devices operating physically or mechanically. Within the nano world, however, chemistry and physics blur.

The concepts we set forth in this paper and the more detailed algorithm we provide are intended to resolve this legal and scientific dilemma. The four pillars of chemicality, singularity, causality, and locality serve as the basis for differentiating chemistry and physics, at least for the purposes of this regulatory framework. As implemented in the detailed algorithm, this proposal answers the mode of action question in a way that satisfies the statutory purpose, meets basic scientific knowledge, and, perhaps most importantly, provides appropriate protection to public health.

Acknowledgments Preparation of this article was supported by National Science Foundation (NSF) grant \#0608791, "NIRT: Evaluating Oversight Models for Active Nanostructures and Nanosystems: Learning from Past Technologies in a Societal Context" (Principle Investigator: S.M. Wolf; Co-PIs: E. Kokkoli, J. Kuzma, J. Paradise, and G. Ramachandran). The views expressed are those of the authors and do not necessarily reflect the views of NSF. The authors wish to extend special thanks to Saurabh Anand for his invaluable assistance in the underlying research and assessment of differing approaches to this issue.

\section{References}

74 Cong. Rec. 4811-12 (April 2, 1935) (statements of Senator Clark)

121 Cong. Rec. 10688, 10690 (April 17, 1975) (testimony by Joseph B. Davis, Director, Scientific Review of the HEW Office of Medical Devices in remarks of Sen. Nelson)

Chevron U.S.A., Inc. v. National Resources Defense Council, Inc., 467 U.S. 837 (1984)

Eells E (1991) Probabilistic causality. Cambridge University Press, New York, NY

Food and Drug Administration (FDA) (1997) Reauthorization of FDA's Medical Device Program. Statement of Michael Friedman before the House Committee on Commerce, 
Subcommittee on Health and Environment. http://www. fda.gov/NewsEvents/Testimony/ucm114951.htm. Accessed 24 Nov 2010

Food and Drug Administration (FDA) (2009) Council for responsible nutrition annual symposium for the dietary supplement industry. Remarks of Joshua M. Sharfstein. http://www.fda.gov/NewsEvents/Speeches/ucm187640.htm. Accessed 24 Nov 2010

Fodor J (1990) Why there still has to be a language of thought. In: Artificial intelligence-a source book. Cambridge University Press, Cambridge

Hutt PB, Merrill RA, Grossman L (2007) Food and drug law: cases and materials. Foundation Press, New York, NY

Lombardi O, Labarca M (2005) The ontological autonomy of the chemical world. Found Chem 7:125-148

Merriam-Webster's Medical Dictionary s.v. "chemistry" (2007) Merriam-Webster, Inc., Springfield, MA

Merrill RA (1996) The architecture of government regulation of medical products. Virginia Law Rev 82:1753-1866

Neurath O (2000) Physicalism. In: Lindberg JJ (ed) Analytic philosophy: beginnings to the present. McGraw-Hill, New York, NY
Nye MJ (1993) From chemical philosophy to theoretical chemistry: dynamics of matter and dynamics of disciplines, 1800-1950. University of California Press, Berkeley, CA

Rai M, Yadav A, Gade A (2009) Silver nanoparticles as a new generation of antimicrobials. Biotechnol Adv 27:76-83

Singh M, Singh S, Prasad S, Gambhir IS (2008) Nanotechnology in medicine and antibacterial effect of silver nanoparticles. Dig J Nanomater Biostruct 3:115-122

Senate Report 361, 74th Cong., 1st sess. (1935)

Senate Report 493, 73rd Cong., 2d Session (1934)

Senate Report 513, 101st Cong., 2d Session (1990)

Sober E (2000) Philosophy of biology. Westview Press, Boulder, CO

United States v. Bacto-Unidisk, 394 U.S. 784 (1969)

Webster's New International Dictionary of the English Language s.v. "chemistry" (1912) G. \& C. Merriam Company, Springfield, MA 